\title{
Herramientas Manuales Vibrátiles y Guantes Anti-Vibrátiles
}

\section{VIBRATING HAND TOOLS AND ANTI-VIBRATING GLOVES}

Rigoberto Quinchía', César G. Lizarazo S², Luis A. Saavedra Robinson², Sildrey Upegui Rincon², Juliana Guarguati Ariza²

1. Centro para la Prevención de Riesgos Profesionales, Medellín, Colombia.

2. Departamento de Ingeniería Industrial, Pontificia Universidad Javeriana, Bogotá, Colombia.

\section{RESUMEN}

Objetivo: Determinar el impacto causado por el tipo de herramienta y tipo de guante sobre la transmisibilidad de la vibración mano-brazo. Métodos: Se realizó un diseño experimental a trabajadores del sector de hidrocarburos, con el objetivo de identificar la transmisibilidad global de los guantes en diferentes actividades. Las mediciones se realizaron a través de dos acelerómetros: uno instalado en la máquina y otro en la palma de la mano. La transmisibilidad fue calculada en el rango de frecuencias $0-2 \mathrm{kHz}$, para cinco tipos de herramientas y 3 tipos de guantes. Finalmente, se compararon los tiempos de exposición a los que puede estar expuesto el trabajador con el uso de los guantes.

Resultados: Se identificó la efectividad de los guantes para proteger a los operarios, permitiéndoles trabajar un mayor número de horas por día. El análisis estadístico evidencia que la variable tipo de herramienta tiene un efecto significativo sobre la transmisibilidad, mientras que el tipo de guante no presenta efecto estadístico alguno. El estudio presenta los porcentajes de aumento de tiempos de exposición y el estudio de las transmisibilidades.

Conclusiones: Los tres tipos de guantes evaluados mostraron tener un desempeño eficaz como atenuadores de la vibración. El aumento del tiempo de exposición permitido al usar guantes es muy significativo para todas las herramientas.

(Quinchía R, Lizarazo C, Saavedra L, Upegui S, Guarguati J, 2018. Herramientas Manuales Vibrátiles y Guantes Anti-Vibrátiles. Cienc Trab. Sep-Dic; 20 [63]: 137-144).

Palabras clave: VIBRACIÓN, TRANSMISIBILIDAD, VIBRACIÓN MANOBRAZO, GUANTES ANTI-VIBRANTES, HERRAMIENTAS MANUALES.

\section{ABSTRACT}

Objective: Determine the impact caused by the type of tool and type of glove on the transmissibility of the hand-arm vibration.

Methods: An experimental design was carried out for workers in the hydrocarbon sector, in order to identify the global transmissibility of gloves in different activities. The measurements were made through two accelerometers: one installed in the machine and the other in the palm of the hand. The transmissibility was calculated in the frequency range $0-2 \mathrm{kHz}$, for five types of tools and 3 types of gloves. Finally, the exposure times to which the worker may be exposed with the use of gloves were compared.

Results: The effectiveness of the gloves was identified to protect the workers, allowing them to work a greater number of hours per day. The statistical analysis shows that the variable type of tool has a significant effect on transmissibility, while the type of glove does not have any statistical effect. The study presents the percentages of increase in exposure times and the study of transmissibilities.

Conclusions: The three types of gloves evaluated showed an effective performance as vibration attenuators. The increase of the exposure time allowed when wearing gloves is very significant for all tools.

Key words: VIBRATION, TRANSMISSIBILITY, HAND-ARM VIBRATION, ANTI-VIBRATION GLOVES, HAND TOOLS.

\section{INTRODUCCIÓN}

Las herramientas portátiles rotativas exponen a los trabajadores a vibraciones periódicas, aleatorias y transitorias que pueden causar riesgos para la salud, incomodidad o una influencia negativa en el desempeño de los trabajadores. Quienes operan estas

\section{Correspondencia / Correspondence:}

Sra. Juliana Guarguati Ariza

Carrera 7 \# 42-27, Bogotá, Colombia

Tel.: +57 (314) $2463-4160$

e-mail: jguarguaty@javeriana.edu.co

Recibido: 10 de agosto de 2018 / Aceptado: 12 de noviembre de 2018 herramientas están expuestos por varias horas durante el día dependiendo de sus ocupaciones. La exposición prolongada a vibraciones por herramientas manuales puede estar asociada a patologías que afectan primordialmente el sistema vascular y neurológico de las personas, como el síndrome del dedo blanco y daños en las articulaciones y músculos de la mano y muñeca. ${ }^{1,2}$ Estas exposiciones prolongadas son frecuentes en trabajadores que emplean maquinaria manual, ya sea neumática o eléctrica durante sus laborales diarias de trabajo. Como estrategia de control contra este factor de riesgo se ha implementado el uso de guantes anti-vibrátiles para los trabajadores, a fin de reducir la intensidad de la vibración transmitida a la parte del cuerpo en contacto, en este caso mano-brazo. Los guantes anti-vibrátiles generalmente proveen una mayor protección en el rango de frecuencias medias. ${ }^{3-5}$ Este criterio es de particular interés para la selección del tipo de guante, ya que se debe buscar que atenúe en 
el rango de frecuencias más cercano a la frecuencia de operación de la herramienta a utilizar para poder asegurar una adecuada protección del trabajador.

La exposición ocupacional a vibraciones mano-brazo ha sido abordada en varios estudios con el objetivo de mejorar el entendimiento de este riesgo y proponer medidas de control para el mismo. ${ }^{5-18}$ Una investigación sobre las características de la vibración transmitida a la muñeca y al codo proveniente de herramientas de impacto manuales sugiere que la medición de vibración en la muñeca es conveniente para monitorear los cambios vibratorios en las herramientas y evaluar la efectividad de los dispositivos anti-vibratorios. ${ }^{6}$ Por otro lado, estudios sobre medidas de control como el desarrollado por De Capua et $\mathrm{al}^{7}$ han propuesto la instalación de un medidor permanente de vibración que active una alarma en caso que el trabajador esté recibiendo una exposición mayor a la permitida. ${ }^{7}$

De igual forma, se evidencian estudios sobre la medición y evaluación de la vibración a nivel ocupacional. Bovenzi8 encontró que las mediciones sin ponderar presentaban mayor correlación con la evolución de las patologías, por lo que el estudio recomienda aumentar los pesos de ponderación para las medias y altas frecuencias al evaluar la vibración mano-brazo. ${ }^{8} \mathrm{~A}$ su vez, Hewitt ${ }^{11}$ identifica factores relevantes que pueden afectar la transmisibilidad de los guantes, tales como los rangos de frecuencia, diferencias de transmisibilidad entre la palma y los dedos, la fuerza aplicada y las características físicas de la persona. $^{11}$

Un estudio sugiere un método alternativo para evaluar la efectividad de los guantes anti-vibratorios, en el cual se concluye que la eliminación del adaptador no afecta la tendencia en la transmisibilidad para las bajas frecuencias, aunque se afectan los valores para las medias y altas frecuencias. ${ }^{12}$ Finalmente, Mahbub et al ${ }^{15}$ estudian la influencia de los guantes anti-vibratorios midiendo el flujo sanguíneo de los dedos. El estudio sugiere que el uso de guantes muestra un cambio considerable en el flujo sanguíneo solo para vibraciones de altas frecuencias. ${ }^{15}$

En este artículo se presenta un diseño experimental para determinar el impacto causado por tipo de herramienta y tipo de guante sobre la transmisibilidad de la vibración, con el objeto de determinar el nivel de efectividad de los guantes anti-vibrátiles para proteger a los trabajadores durante una jornada de trabajo.
A su vez, analiza las características de las herramientas manuales vibrátiles y el impacto que genera el uso de los guantes antivibrátiles. Finalmente, este estudio compara los tiempos de exposición a los que puede estar expuesto el trabajador con el uso o no de los guantes y el aumento de productividad que este puede generar a las empresas.

\section{MÉTODOS}

Se llevó a cabo un diseño experimental en el cual se realizaron mediciones de vibración mano-brazo a trabajadores del departamento de mantenimiento de una planta del sector de hidrocarburos. Se utilizaron herramientas disponibles en el área, las cuales fueron manipuladas por los trabajadores durante el experimento, con el objetivo de lograr que las evaluaciones generaran la mayor representatividad posible. Los accesorios de las herramientas y las prácticas de manufactura se controlaron para garantizar la reproducibilidad de las mediciones en bancos de prueba concertados para estas pruebas. Los trabajadores fueron escogidos de forma aleatoria según disponibilidad durante el turno de trabajo.

Los factores estudiados fueron el tipo de guante y tipo de herramienta. Se emplearon 3 tipos de guantes: Proflex, Decade y Air Glove (certificados en ISO 10819) y 5 tipos de herramientas; 3 eléctricas: pulidora, motor-tool y esmeril; y dos herramientas neumáticas: llave de impacto y martillo neumático. ${ }^{3}$ Las Figuras 1 y 2 muestran las herramientas utilizadas en el estudio y los diferentes tipos de guantes anti-vibrátiles certificados que fueron utilizados.

La variable respuesta del diseño experimental es la transmisibilidad global de los guantes, y fue evaluada en el rango $0-2 \mathrm{kHz}$. Adicionalmente, se estudió el comportamiento de la transmisibilidad en tres sub-rangos diferentes de frecuencias (bajo: 0-31,5 Hz, medio: 31,5-200 Hz y alto: 200-2000 Hz). Para las mediciones de vibración se usaron dos acelerómetros, los cuales registraron las vibraciones para cada actividad: uno instalado en la máquina y otro en la palma de la mano (al interior del guante anti-vibrátil cerca al tercer metacarpiano con una abrazadera tipo anillo). Los dos acelerómetros se posicionaron acorde a la norma, teniendo presente que las direcciones de los ejes entre los acelerómetros fueran coincidentes.

Figura 1.

Tipos de herramientas utilizadas en el diseño experimental.

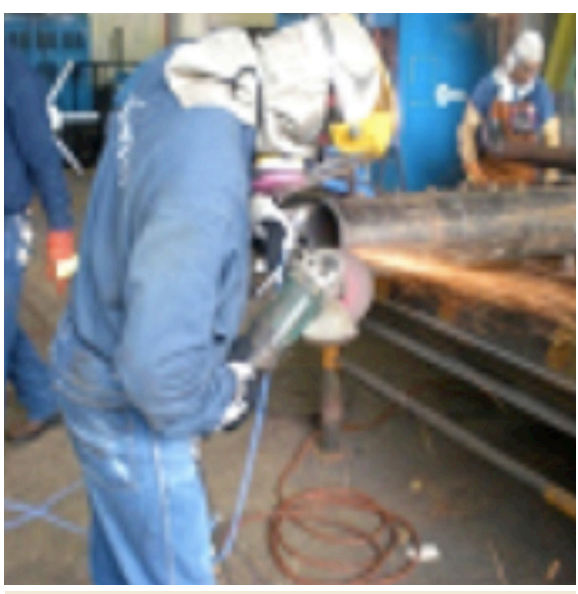

Pulidora

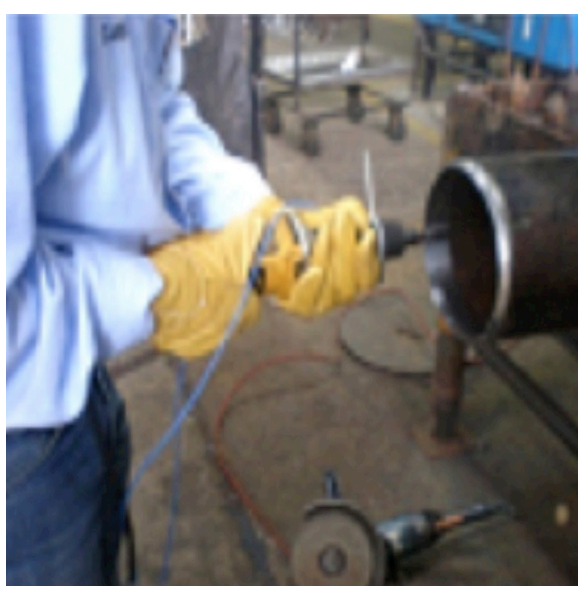

Motor tool

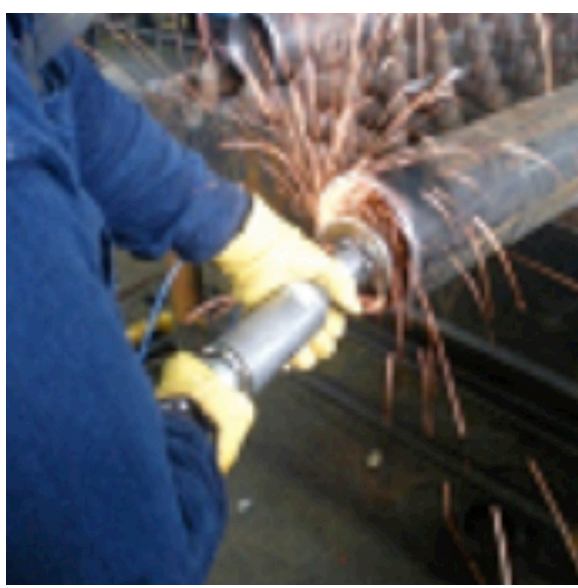

Esmeril 
Figura 1.

Tipos de herramientas utilizadas en el diseño experimental.

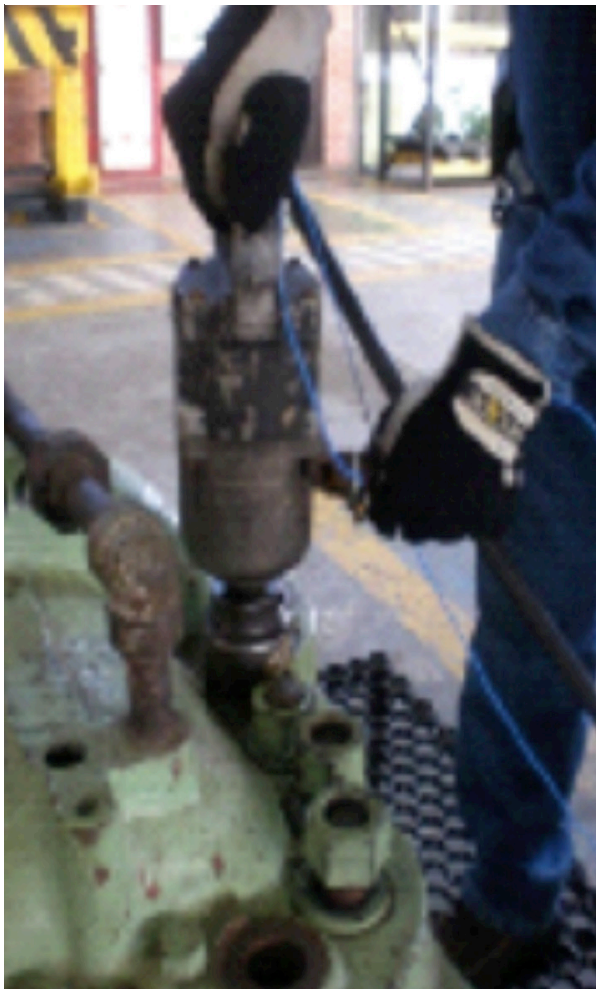

Llave de Impacto

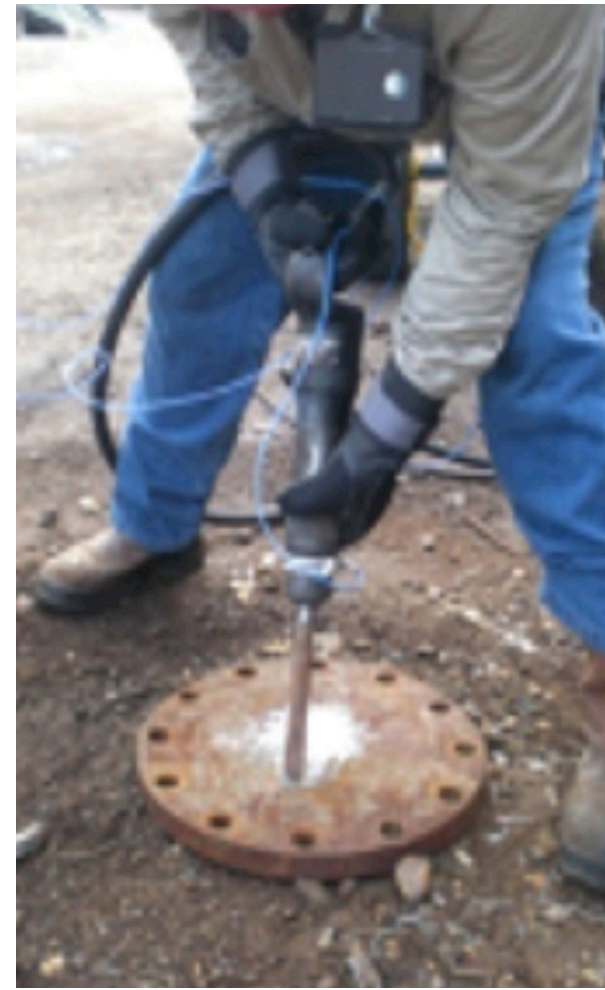

Martillo Neumático

con el fin de evaluar el efecto aleatorio de las mediciones y la efectividad sobre las variables controladas para la reproducibilidad de las medidas. En total se recolectaron 15 formatos de mediciones, para 5 herramientas y 3 tipos de guantes. Los datos de cada formato corresponden a las tres réplicas tomadas en cada medición.

Finalmente, con el fin de asegurar la menor variabilidad posible para las réplicas realizadas, se prepararon 3 bancos de pruebas, en los cuales fue desarrollado el diseño experimental. En el primer banco se empleó un tubo metálico para realizar medidas con herramientas eléctricas como la pulidora, esmeril y el motor-tool. En el segundo banco de prueba se realizaron pruebas con la pistola neumática y en el tercer banco de prueba se desarrollaron pruebas con el martillo neumático. Ver Figura 3.

\section{Análisis de Datos}

Para evaluar la exposición a las vibraciones se dio prioridad a las frecuencias que tenían mayor efecto sobre las partes del cuerpo humano que recibieron la vibración; es decir, que se realizó una ponderación por frecuencia. Adicionalmente, se calculó la resultante

Para las mediciones se empleó un equipo marca Quest Technologies modelo VI 410, el cual permite el uso de acelerómetros triaxiales y cumple con los referentes estándares ISO 8041 y ISO 61672$1.19,20 \mathrm{Al}$ inicio y final de las mediciones para cada herramienta se realizaron mediciones de verificación (calibración secundaria) con el shaker para confirmar la correcta calibración de los medidores.

Para cada herramienta se evaluaron los espectros de vibración. Se realizaron tres réplicas de las mediciones para cada herramienta que considera la vibración en un sistema de referencia triaxial.

Para la medición de las vibraciones mano-brazo se tuvieron presentes los siguientes aspectos:

- Definir el rango de frecuencia cubierto por las bandas de octava que ayudaran a estimar el efecto adverso de las vibraciones transmitidas. En el caso de mano-brazo es 0-2 kHz.

- Definir el filtro de ponderación frecuencial para cada eje, para dar prioridad a las bandas de frecuencias que tengan mayor efecto adverso. Para mano-brazo se utiliza el filtro Wh.

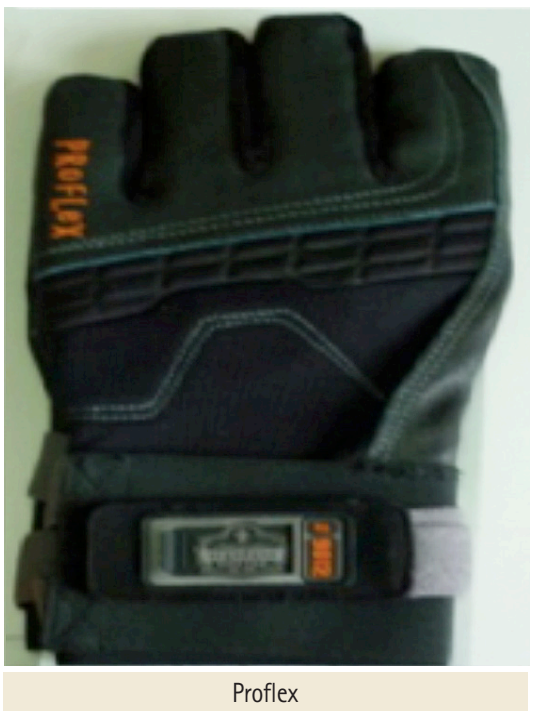

Figura 2.

Tipos de guantes empleados en el diseño experimental.

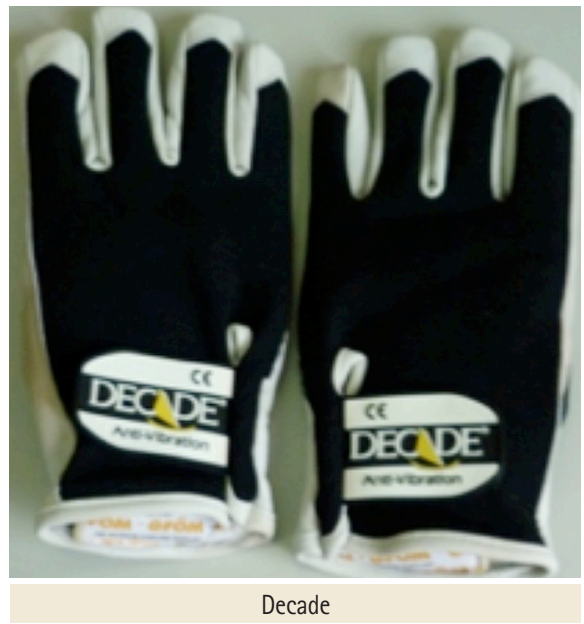

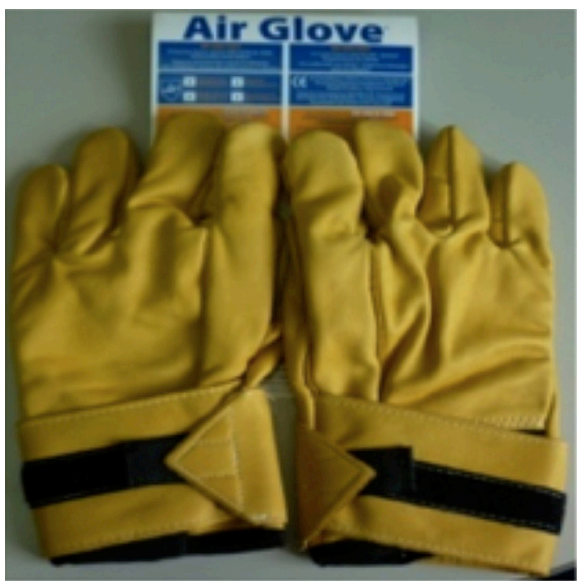

Air Glove 
Figura 3.

Bancos de prueba utilizados.

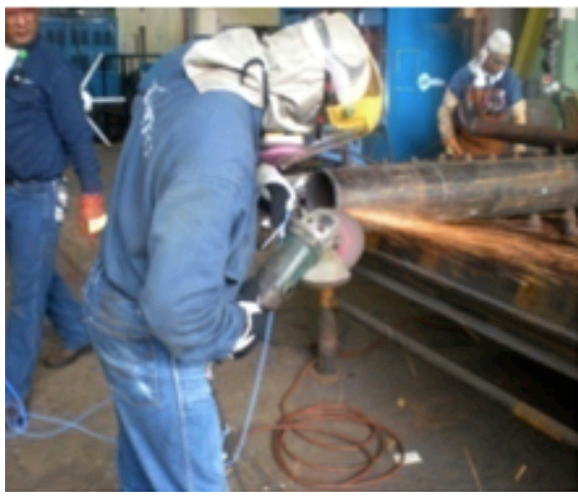

(A)

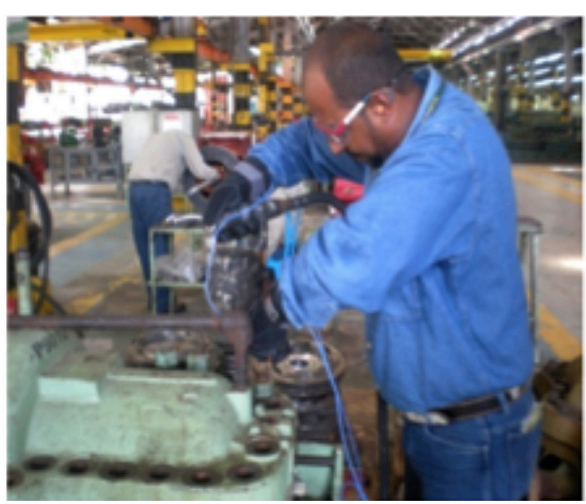

(B)

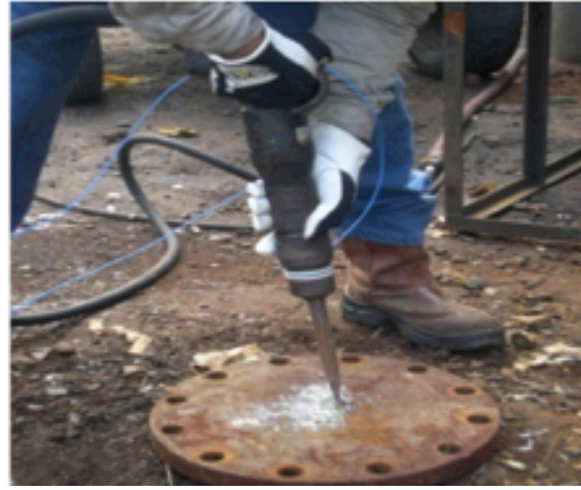

(C)
- Realizar la ponderación de frecuencias con el filtro adecuado (Wh) para cada banda de octava (subíndice i) en cada eje (subíndice j), para obtener las aceleraciones en cada eje awx, awy y awz acorde a la fórmula:

$$
\mathrm{a}_{w j}=\left[\sum_{i}\left(\mathrm{w}_{i} \mathrm{a}_{i j}\right)^{2}\right]^{1 / 2}
$$

- Calcular la resultante de la aceleración teniendo presente los tres ejes. Cada eje puede tener una importancia diferente por lo que se utilizan los coeficientes $\mathrm{kj}$. En el caso mano-brazo kx $=\mathrm{ky}$ $=\mathrm{kz}=1$

$$
a_{w}=\sqrt{\left(k_{x} a_{w X}\right)^{2}+\left(k_{y} a_{w y}\right)^{2}+\left(k_{z} a_{w z}\right)^{2}}
$$

Las aceleraciones medidas son características de un tipo de actividad. En un oficio se pueden estar sometidos a diferentes actividades por diferentes periodos de tiempo durante la jornada de trabajo; en estos casos se debe evaluar cada ciclo con su respectivo período de exposición y sumar los efectos con la expresión descrita a continuación:

$$
\mathrm{A}(8)=\sqrt{\frac{1}{T_{o}} \sum_{i=1}^{n} \mathrm{a}_{\text {resultante }}^{2} T_{i}}
$$

Para el estudio se tomó un tiempo de referencia de $\mathrm{T} 0=8 \mathrm{~h}$ y Ti como el tiempo de exposición por cada actividad.

El valor A (8) fue comparado con los valores de nivel de acción $(E A V=2.5 \mathrm{~m} / \mathrm{s})$ y el valor límite permisible $(E L V=5 \mathrm{~m} / \mathrm{s})$, establecido por la norma de la Unión Europea, la cual define los rangos bajo, medio o alto de exposición para los oficios que utilizan herramientas manuales vibrátiles. ${ }^{21}$

Una vez calculada la intensidad de la vibración para cada herramienta, se procedió a calcular el tiempo requerido para llegar al valor de nivel de acción (EAV: Exposure Action Value) y valor límite (ELV: Exposure Limit Value), fórmulas (4) y (5):

$$
\begin{aligned}
& E A V=8\left(\frac{2.5}{a_{w}}\right)^{2} \\
& E A V=8\left(\frac{5}{a_{w}}\right)^{2}
\end{aligned}
$$

La transmisibilidad puede ser medida en diferentes rangos de frecuencia. Para el análisis en este estudio se midió la transmisibilidad total, TT, en todo el rango de frecuencias (0-2 kHz), la transmisibilidad en el rango A (0-31.5 Hz): TA, la transmisibilidad en el rango B (31.5-200 Hz): TB, y la transmisibilidad en el rango C (200-2000 Hz): TC. Estos valores fueron estudiados con el fin de conocer los comportamientos en las bajas, medias y altas frecuencias.

\section{RESULTADOS}

\section{Caracterización espectral de las herramientas}

Los datos de las amplitudes para cada banda frecuencial fueron tomados directamente del equipo Quest VI-410. En la Figura 4, se observan los espectros de frecuencias vibratorias de las herramientas eléctricas evaluadas; estos espectros evidencian que las tres herramientas eléctricas poseen la mayoría de los componentes en las altas frecuencias (superiores a $200 \mathrm{~Hz}$ ), hecho esperado dado que giran a altas rpm. Por el contrario, el espectro de frecuencias vibratorias de las herramientas neumáticas presentan las amplitudes homogéneamente distribuidas en todo el espectro de frecuencias, presentando un comportamiento similar al de un ruido blanco. El espectro de la llave neumática tiene repartidas las amplitudes de las principales componentes frecuenciales en los extremos, mostrando ausencia de niveles significativos de vibración en las frecuencias medias.

\section{Transmisibilidad, EAV y ELV para los casos estudiados}

En la tabla 1 se muestra un ejemplo de los resultados obtenidos. En este caso, se muestran los resultados de las aceleraciones para cada réplica de la llave de impacto con el guante 1 (Proflex $\left.{ }^{\circledR}\right)$ y sin el guante. Siguiendo la nomenclatura awh para los resultados sin guante y awg con guante. Este procedimiento de cálculo se realizó para cada herramienta y cada tipo de guante. Los cálculos fueron realizados para 3 rangos de frecuencias, a fin de observar el comportamiento de la transmisibilidad en cada rango. De igual forma, se calcularon los EAV y ELV para cada uno de las mediciones.

\section{Análisis estadístico}

\begin{tabular}{|c|c|c|c|c|c|c|}
\hline & \multicolumn{6}{|c|}{ Llave de Impacto - Guante Proflex ${ }^{\circledR}$} \\
\hline \multirow{4}{*}{$\Pi$} & \multicolumn{2}{|c|}{ Réplica 1} & \multicolumn{2}{|c|}{ Réplica 2} & \multicolumn{2}{|c|}{ Réplica 3} \\
\hline & awh & awg & awh & awg & awh & awg \\
\hline & 17,35 & 6,25 & 12,15 & 8,20 & 9,36 & 6,37 \\
\hline & \multicolumn{2}{|c|}{0,360} & \multicolumn{2}{|c|}{0,675} & \multicolumn{2}{|c|}{0,681} \\
\hline
\end{tabular}

Se realizó un análisis de varianza (ANOVA) para entender el efecto de las variables tipo de guante y tipo de herramienta sobre la

Tabla 1.

Resultados llave de impacto - guante Proflex ${ }^{\circledR}$. 
Figura 4.

Espectros de frecuencia vibratoria para las herramientas eléctricas y neumáticas.
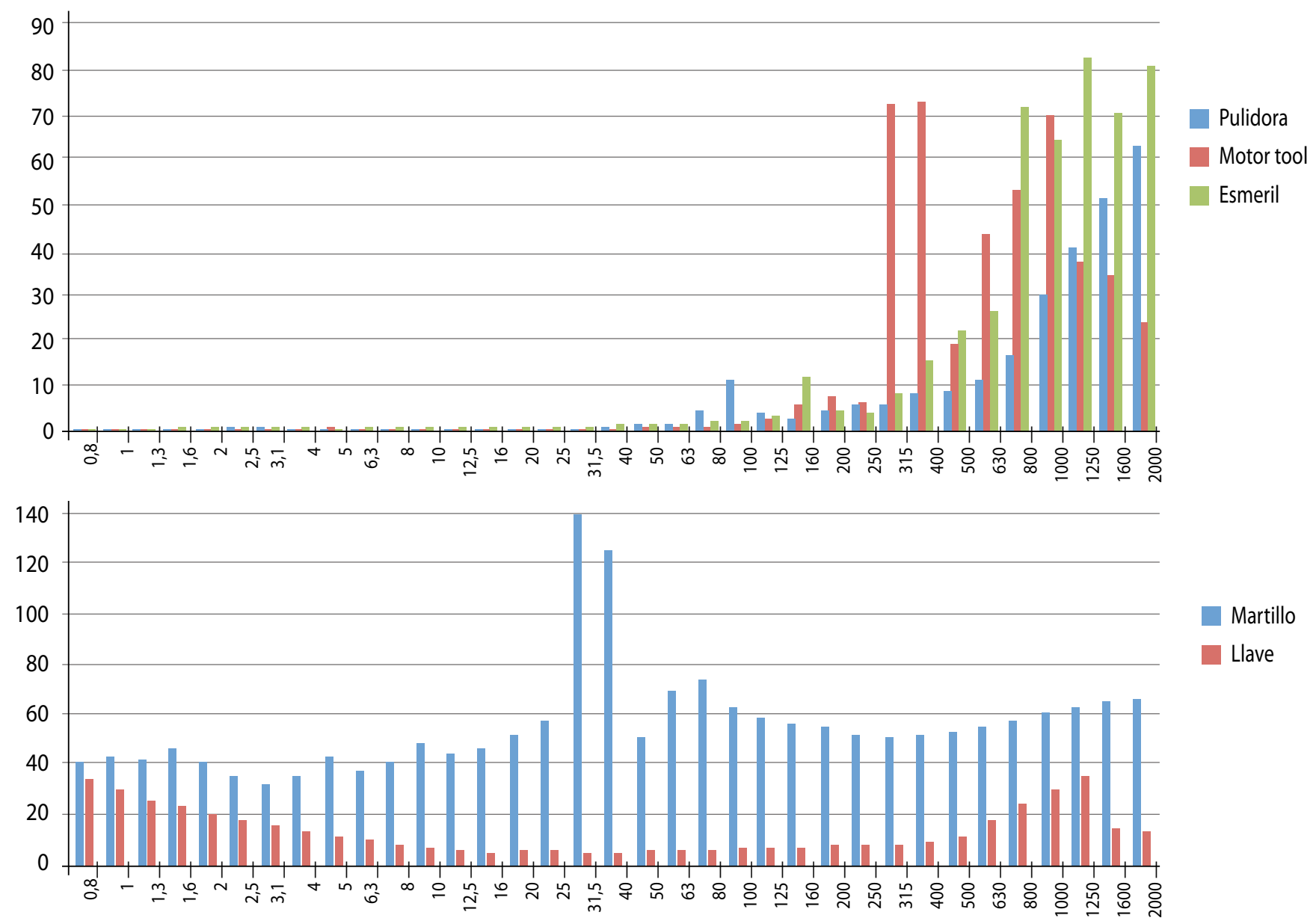

variable respuesta: transmisibilidad total TT. El análisis permite observar el efecto estadísticamente significativo de cada variable. Para el análisis se utilizó un criterio de significancia de 0,05, el cual asegura que la probabilidad (p) de obtener un resultado cierto a pesar de ser falso es de sólo 5\%. Los resultados del análisis evidencian que la variable "tipo de herramienta", tiene un efecto estadísticamente significativo sobre la transmisibilidad total, ya que los valores de $\mathrm{p}<0.05$. Por el contrario, la variable tipo guante y las interacciones no muestran efecto estadísticamente significativo ( $\mathrm{p}>0.05)$. Ver Tabla 2.

Tabla 2.

Análisis de Varianza para TT.

\begin{tabular}{|c|c|c|c|c|c|}
\hline Fuente & $\begin{array}{l}\text { Suma de } \\
\text { cuadrados }\end{array}$ & $\begin{array}{l}\text { Grados de } \\
\text { libertad }\end{array}$ & $\begin{array}{l}\text { Cuadrado } \\
\text { medio }\end{array}$ & Relación F & Valor de $\mathrm{P}$ \\
\hline \multicolumn{6}{|c|}{ Efectos Principales } \\
\hline A: Guantes & 0,0477 & 2 & 0,0238 & 1,97 & 0,157 \\
\hline B: Herramienta & 1,3162 & 4 & 0,3290 & 27,19 & 0,000 \\
\hline \multicolumn{6}{|l|}{ Interacciones } \\
\hline$A B$ & 0,07347 & 8 & 0,0092 & 0,76 & 0,640 \\
\hline Residuo & 0,3630 & 30 & 0,0121 & & \\
\hline Total & 1,8003 & 44 & & & \\
\hline
\end{tabular}

En la Figura 5 se evidencian las medias de transmisibilidad obtenidas para cada tipo de guante y cada tipo de herramienta, respectivamente. En el caso de la variable tipo de guante, la transmisibilidad más baja fue reportada para el guante 2 (Decade ${ }^{\circledR}$ ), es decir que, aparentemente, este tipo de guante transmite en menor intensidad la vibración al operador. Sin embargo, la variabilidad de cada guante se traslapa con los otros y, por tanto, no hay efecto significativo por el tipo de guante utilizado. Es decir que, en principio, no existe gran diferencia en utilizar cualquiera de los tres tipos de guantes.

En el caso de la variable tipo de herramienta, para el martillo, el motor-tool, el esmeril y la pulidora, la atenuación fue muy buena debido a que la transmisibilidad fue baja. Esto quiere decir que la utilización de los guantes en esas cuatro herramientas es especialmente eficaz. Para la llave neumática, la transmisibilidad estuvo alrededor del 60\%, lo que indica que el guante de igual forma resulta útil, aunque en menores proporciones que las cuatro herramientas anteriormente mencionadas.

Adicionalmente, se realizaron análisis similares para cada uno de los tres sub-rangos de frecuencias: baja, media y alta, con el fin tener un entendimiento mayor sobre la transmisibilidad total. Los sub-rangos de frecuencias fueron clasificados de la siguiente forma: frecuencia baja de $0-31.5 \mathrm{~Hz}$, media de $31.5-200 \mathrm{~Hz}$ y alta de $200-2000 \mathrm{~Hz}$. 
Figura 5.

Resultados de transmisibilidad por tipo de guante y tipo de herramienta.

Transmisibilidad Totales :

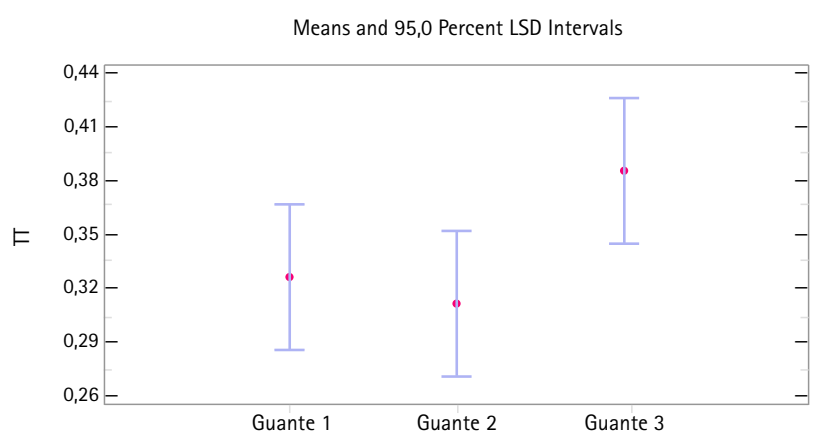

Transmisibilidad Bajas Frecuencias :

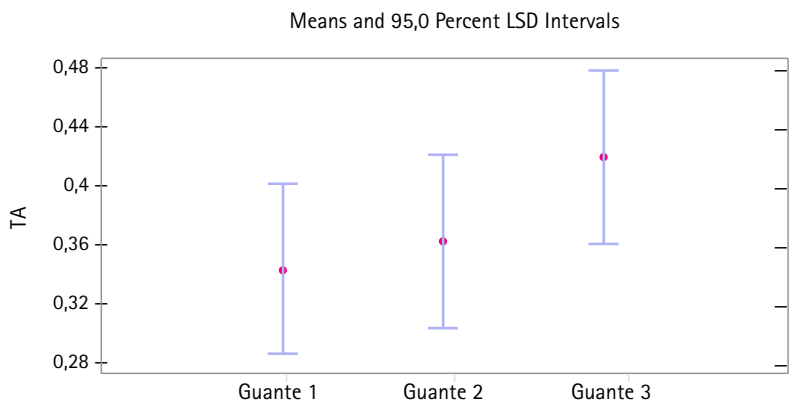

Transmisibilidad Medias Frecuencias :

Means and 95,0 Percent LSD Intervals

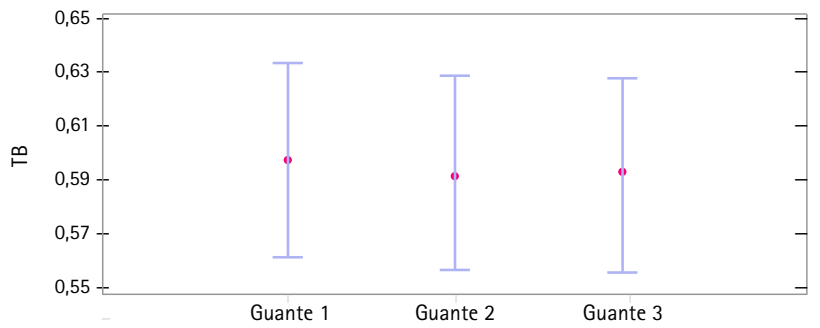

En el análisis para las frecuencias bajas, la variable "tipo de herramienta" tuvo un efecto estadísticamente significativo sobre la transmisibilidad a baja frecuencia (TA), mientras que ni el tipo de guante ni sus interacciones tuvieron efecto. La Figura 5 muestra las medias de transmisibilidad obtenidas para cada tipo de guante y cada tipo de herramienta. Para este rango de frecuencias, la medida de transmisibilidad de los guantes 1 y 2 estuvieron muy cercanas, aunque para este rango, el guante 1 tuvo una transmisibilidad un poco inferior que el guante 2 . El guante 3 continúa siendo el guante con mayor transmisibilidad, es decir, el guante que menos atenúa la vibración. Por otro lado, la transmisibilidad de las bajas frecuencias para el motor-tool y la llave son especialmente altas.

En el análisis para las frecuencias medias, solo la variable "tipo de herramienta" tuvo efecto estadísticamente significativo sobre la transmisibilidad a media frecuencia (TB), mientras que ni el tipo de guante, ni las interacciones tuvieron efecto sobre la transmisibi-
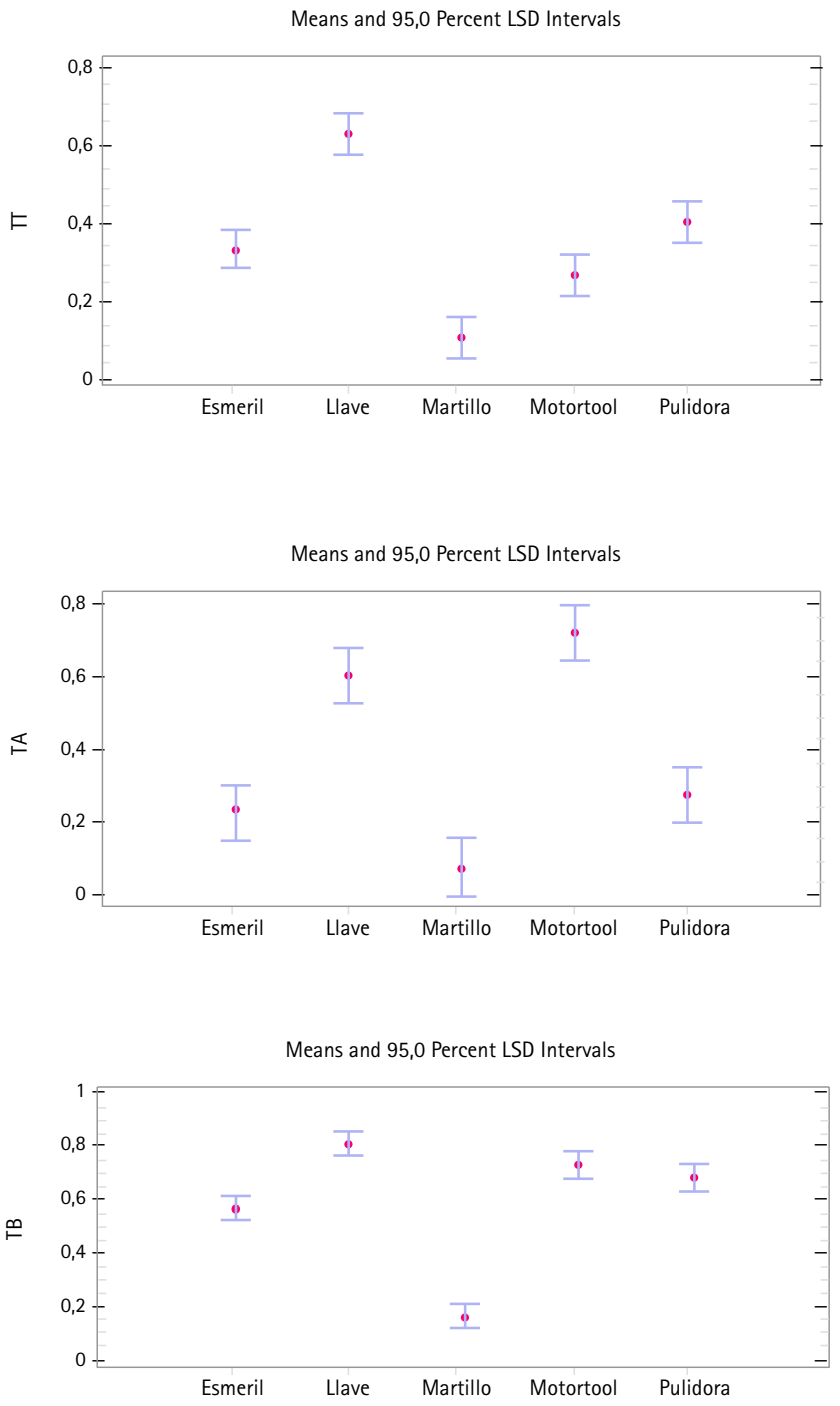

lidad. Esto quiere decir que para las medias frecuencias también es indiferente el tipo de guante que sea seleccionado. La Figura 5 muestra las medias de transmisibilidad obtenidas para cada tipo de guante y cada tipo de herramienta. Para este rango de frecuencias, en la figura se observa que los guantes 2 y 3 presentan las transmisibilidades más bajas; sin embargo, las varianzas son tan grandes que sus valores se traslapan, por lo que se podría concluir que no hay un efecto estadísticamente significativo del tipo de guante sobre la transmisibilidad de las medias frecuencias. De igual forma, se observa que el comportamiento de las transmisibilidades para las diversas herramientas en frecuencias medias es muy parecido al comportamiento de las frecuencias bajas.

Para el análisis de las frecuencias altas, las variables "tipo de herramienta”, "tipo de guante" y las interacciones no tuvieron efecto estadísticamente significativo sobre la transmisibilidad a altas frecuencia (TC). Esto quiere decir que, para este rango de frecuencias, el estudio evidencia que es indiferente el tipo de guante que 
se seleccione y es indiferente la herramienta que se esté usando. Cabe resaltar que en estudio se utilizó el filtro Wh para las mediciones mano-brazo acorde a la norma ISO 5349. Este filtro resta mucha importancia a las altas frecuencias (sus multiplicadores a las altas frecuencias son casi cero); por ende, la variable tipo de herramienta tampoco tuvo efecto estadísticamente significativo en las altas frecuencias. Por no existir efecto estadísticamente significativo de ninguno de las variables ni de las interacciones, no se ilustran los comportamientos de las medias.

\section{Análisis de mejora de los tiempos de exposición}

Uno de los análisis más relevantes en este estudio es cómo varían los tiempos de exposición al utilizar los guantes para las diversas herramientas de trabajo. En este caso, se estudian los cambios en los EAV y en los ELV al utilizar guantes anti-vibrátiles. En la Tabla 3 se ilustra el porcentaje de aumento de los tiempos para alcanzar el límite de acción diario cuando se utilizan los guantes antivibrátiles. Por ejemplo, sin el uso de los guantes, un trabajador puede hacer uso de la herramienta esmeril hasta por 3,24 horas (tiempo en que se alcanza el límite de acción), mientras que, con el uso de los guantes el trabajador puede aumentar el tiempo de trabajo con el esmeril a 8 horas. En la tabla se muestran los resultados EAVH para los límites de acción sin el uso de guantes y EAVG con el uso de los mismos. De igual forma, se ilustra el cambio de los tiempos necesarios para llegar al límite máximo de exposición. Se observa, por ejemplo, que la llave neumática tenía un ELV sin guantes de 1,52 horas y pasa a 4 horas por la utilización de los guantes. Esto refleja un aumento en tiempo de utilización del 164\%.

Tabla 3.

Análisis de la influencia de los guantes en los EAV y ELV.

$\begin{array}{lccccc}\begin{array}{l}\text { Herramienta / } \\ \text { Variable }\end{array} & \text { Esmeril } & \begin{array}{c}\text { Llave } \\ \text { Neumática }\end{array} & \begin{array}{c}\text { Martillo } \\ \text { Neumática }\end{array} & \text { Motor-tool } & \text { Pulidora } \\ \text { EAVH } & 3,24 & 0,38 & 0,003 & 1,48 & 4,05 \\ \text { EAVG } & 8 & 1 & 0,32 & 8 & 8 \\ \% \text { aumento } & 147 & 164 & 10427 & 441 & 98 \\ \text { ELVH } & 8 & 1,52 & 0,012 & 5,92 & 8 \\ \text { ELVG } & 8 & 4,008 & 1,26 & 8 & 8 \\ \% \text { aumento } & 0 & 164 & 10400 & 35 & 0\end{array}$

\section{DISCUSION}

Los tres tipos de guantes evaluados mostraron tener un desempeño eficaz como atenuadores de la vibración para proteger a los trabajadores. Aunque el análisis de varianza no mostró un efecto estadísticamente significativo por el tipo de guante, es importante resaltar que al evaluar las medias se presentaron pequeñas diferencias, las cuales podrían ser relevantes en la toma de decisiones a la hora de escoger alguno de los tres guantes anti-vibrátiles. El guante 2 (Decade $\left.{ }^{\circledR}\right)$ mostró un desempeño superior respecto a la atenuación de las vibraciones, ya que presentó una transmisibilidad total promedio de 0,31 . El guante 1 (Proflex $\left.{ }^{\circledR}\right)$ presentó una transmisibilidad de 0,32, es decir, un 3,2\% mayor respecto al guante 2. El guante 3 (Air Glove) mostró una transmisibilidad de 0,38 , un 22,5\% mayor respecto al guante 2 .

Las herramientas neumáticas poseen una vibración mucho más fuerte que las herramientas eléctricas evaluadas. La transmisibilidad obtenida para el martillo, el motor-tool, el esmeril y la pulidora fueron relativamente bajas, permitiendo concluir que la utilización de guantes anti-vibrátiles para estos tipos de herramientas resulta particularmente útil como medida de protección a los trabajadores.

El estudio permite concluir que el uso de guantes anti-vibrátiles resulta ser una medida efectiva para la protección de trabajadores que emplean herramientas manuales. El aumento del tiempo de exposición permitido al usar guantes es muy significativo para todas las herramientas evaluadas. Para las herramientas eléctricas evaluadas -esmeril, pulidora y motor-tool-, el uso de guantes permite la utilización de estas herramientas por 8 horas diarias de trabajo. De igual forma, los tiempos de exposición para alcanzar los valores de acción, sin guantes, para la llave neumática fue de $0.38 \mathrm{~h}$ (22,8 $\mathrm{min})$ y para el martillo 0,003 h (0,18 min). Al utilizar guantes los tiempos se incrementan notoriamente, a $1 \mathrm{~h}$ y $0,32 \mathrm{~h}$ (19,2 min), respectivamente. El incremento de estos tiempos de trabajo permite a las empresas aumentar la productividad de las mismas sin dejar a un lado la seguridad y salud de los trabajadores, ya que se estaría aumentando el tiempo de exposición, pero con las debidas medidas de protección como lo son los guantes anti-vibrátiles. De igual forma, se debe asegurar no sobrepasar bajo ninguna circunstancia los límites de acción definidos para cada tarea. 


\section{REFERENCIAS}

1. Soto $A D$. Procedimiento de evaluación de riesgos ergonómicos y psicosociales [tesis]. Almeria: Universidad Almería; 2015. p. 50-62.

2. International Organization for Standardization (ISO). ISO 5349-1:2001: Mechanical vibration; Measurement and evaluation of human exposure to hand-transmitted vibration. Part 1: General requirements [online]. Geneva: International Organization for Standardization; 2001 [cited 2017 Nov 10]. Available from: https://www.iso.org/standard/32355.html.

3. International Organization for Standardization (ISO). ISO 10819:2013 Mechanical Vibration and Shock; Hand-Arm Vibration: Measurement and Evaluation of the Vibration Transmissibility of Gloves at the Palm of the Hand [online]. Geneva: International Organization for Standardization; 2013 [cited 2017 Nov 10]. Available from: https://www.iso.org/standard/46313.html.

4. Hewitt S. Triaxial measurements of the performance of anti-vibration gloves. HSE Research Report RR795 [online]. Derbyshire: HSE-HSL; 2010 [cited 2017 Nov 20]. Available from: http://www.hse.gov.uk/research/rrpdf/rr795.pdf.

5. Pinto I, Stacchini N, Bovenzi M, Paddan GS, Griffin MJ. Protection effectiveness of anti-vibration gloves: field evaluation and laboratory performance assessment. Proceedings of the 9th International Conference on Hand-arm Vibration; 2001 Jun 5-8; Nancy, Francia. 2001. Paris: INRS; 2001.

6. Xu XS, Welcome DE, McDowell TW, Warren C, Dong RG. An investigation on characteristics of the vibration transmitted to wrist and elbow in the operation of impact wrenches. Int J Ind Ergonom. 2009; 39(1):174-184.

7. De Capua C, Meduri A, Morello R. A hand-arm vibration meter monitoring the percussion exposure for health risk prevention applications. Proceedings of the IEEE International Workshop of Medical Measurements and Applications; 2009 May 29-30; Cetraro, Italy [online]. New Jersey: IEEE; 2009. p. 45-50.

8. Bovenzi M. A longitudinal study of vibration white finger, cold response of digital arteries, and measures of daily vibration exposure. Int Arch Occup Environ Health. 2010; 83(3):259-272.

9. Hewitt S. Assessing the performance of anti-vibration gloves; A possible alternative to ISO 10819, 1996. Ann Occup Hyg. 1998; 42(4):245-252.

10. Hewitt $S$, Dong RG, Welcome DE, McDowell TW. Anti-vibration gloves? Ann Occup Hyg. 2014; 59(2):127-141.

11. Hewitt S, Dong R, McDowell T, Welcome D. The efficacy of anti-vibration gloves. Acoust Aust. 2016; 44(1):121-127.
12. Dong RG, Rakheja S, McDowell TW, Welcome DE, Wu JZ, Warren C, et al. A method for assessing the effectiveness of anti-vibration gloves using biodynamic responses of the hand-arm system. J Sound Vib. 2005; 282(3-5):11011118.

13. Dong RG, Mc Dowell TW, Welcome DE, Warren C, Wu JZ, Rakheja S. Analysis of anti-vibration gloves mechanism and evaluation methods. J Sound Vib. 2009; 321(1-2):435-453.

14. Dong RG, Welcome DE, Peterson DR, Xu XS, McDowell TW, Warren $C$, et al. Tool-specific performance of vibration-reducing gloves for attenuating palmtransmitted vibration in three orthogonal directions. Int J Ind Ergonom. 2014; 44:827-839.

15. Mahbub MH, Yokoyama K, Laskar MS, Inoue M, Takahashi Y, Yamamoto S, et al. Assessing the influence of anti-vibration glove on digital vascular responses to acute hand-arm vibration. J Occup Health. 2007; 49(3):165-171.

16. Welcome DE, Dong RG, XuXS. The effects of vibration-reducing gloves on finger vibration. Int J Ind Ergonom. 2014; 44(1):45-49.

17. Iriondo MR. Vibraciones mecánicas qué son y cómo se evalúa el riesgo. DYNA. 2007; 82(9):499-506.

18. UK. Health and Safety Executive (HSE). Hand-arm vibration; Schedule 1. In: HSE. Statuory Instruments 2005 No.1093. The control of vibration at work regulations. London: HSE Books; 2005. p. 8-9.

19. International Organization for Standardization (ISO). ISO 8041-1:2017: Human response to vibration; Measuring instrumentation. Part 1: General purpose vibration meters [online]. Geneva: International Organization for Standardization; 2017 [cited 2017 Nov 20]. Available from: https://www.iso. org/standard/70648.html.

20. International Electrotechnical Commission (IEC). International Standard IEC 61672 -1: A Standard for Sound Level Meters [online]. Geneva: International Electrotechnical Commission; 2013 [cited 2017 Nov 11]. Available from: https:// webstore.iec.ch/preview/info_iec61672-1\%7Bed2.0\%7Db.pdf.

21. Comisión Europea. Guia de buenas prácticas no vinculante para la aplicación de la Directiva 2002/44/CE, sobre las disposiciones mínimas de seguridad y de salud relativas a la exposición de los trabajadores a los riesgos derivados de los agentes físicos (vibraciones). Luxemburgo: Oficina de Publicaciones Oficiales de las Comunidades Europeas; 2008. 120 p. 